\title{
Twists of Opposite Handedness on a Scroll Wave
}

\author{
Patricia Dähmlow, ${ }^{1}$ Sergio Alonso, ${ }^{2}$ Markus Bär, ${ }^{2}$ and Marcus J. B. Hauser ${ }^{1, *}$ \\ ${ }^{1}$ Institut für Experimentelle Physik, Abteilung Biophysik, Otto-von-Guericke Universität Magdeburg, \\ Universitätsplatz, 2, 39106 Magdeburg, Germany \\ ${ }^{2}$ Physikalisch-Technische Bundesanstalt, Abbestraße 2-12, 10587 Berlin, Germany
}

(Received 21 December 2012; published 5 June 2013)

\begin{abstract}
The dynamic interaction of scroll waves in the Belousov-Zhabotinsky reaction with a vertically orientated gradient of excitability is studied by optical tomography. This study focuses on scroll waves, whose filaments were oriented almost perpendicular to the gradient. Whereas scroll waves with filaments exactly perpendicular to the gradient remain unaffected, filaments with a component parallel to the gradient develop a twist. Scroll waves with U-shaped filaments exhibit twists starting from both of its ends, resulting in scroll waves whose filaments display a pair of twists of opposite handedness. These twists are separated by a nodal plane where the filament remains straight and untwisted. The experimental findings were reproduced by numerical simulations using the Oregonator model and a linear gradient of excitability almost perpendicular to the orientation of the filament.
\end{abstract}

DOI: 10.1103/PhysRevLett.110.234102

PACS numbers: 05.45.- a, 05.65.+b, 82.40.Ck, 89.75.Kd

Introduction.-Scroll waves occur in three dimensional (3D) excitable media and represent a prominent example for self-organization in systems where reactions interplay with diffusive transport, such as the Belousov-Zhabotinsky (BZ) reaction. Cardiac tissue is another excitable medium where scroll waves play an important role in the generation of cardiac arrhythmias, like ventricular tachycardia [1,2] and fibrillation [3].

A scroll wave can be considered as a stack of rotating spiral waves that are organized by the line connecting the cores. This line is called the filament, around which the scroll wave rotates [4-6]. The motion of scroll waves is enslaved to the dynamics of the filament. A straight scroll wave is stable if the filament has positive tension and wave turbulence is obtained with negative filament tension [6,7]. The internal organization of such complex three-dimensional waves can be studied using the BZ reaction where scroll waves can either be induced or formed spontaneously [4]. This reaction permits the visualization of arrhythmialike chemical waves, which are difficult to visualize in the heart muscle [8].

Wave propagation in the heart depends on fiber orientation in the myocardium, which changes from epicardium to endocardium. Furthermore, heart tissue is highly heterogeneous and anisotropic [9], leading to a propagation velocity of the excitable waves, which depends on the tissue anisotropy [10,11]. In such inhomogeneous systems, the orientation of scroll wave filaments may be complex depending on both the anisotropy and heterogeneity of the tissue. Interestingly, scroll wave filaments in anisotropic media were shown to describe geodesics in a curved space $[12,13]$, and the equations governing their behavior resemble closely those known from cosmic strings in the universe [13].

The most studied case of scroll wave dynamics under inhomogeneous conditions is a single scroll wave oriented parallel to a spatial gradient of excitability. Under such conditions, the dynamics of the scroll wave is affected by a gradient of excitability and the scroll wave may become unstable [6,14-18] and twisted [15,19,20]. The twist propagates along the filament. Experimental realizations of gradients in a 3D BZ medium were studied by using gradients of temperature [17], illumination intensity [21], reactant concentrations [18,22], and electric current $[23,24]$. Sufficiently large gradients may induce a transition of the filament motion from a rigidly rotating to a meandering trajectory [25].

Gradients of excitability may also exert additional effects on 3D structures. Depending on the orientation of the scroll ring (i.e., a scroll wave with a circular filament), the gradient may lead to its reorientation $[23,24,26]$.

In contrast to scroll waves influenced by gradients oriented in parallel to the filament, much less is known for the dynamics of scroll wave filaments oriented perpendicularly to the gradient. Therefore, in the present study a vertical gradient of excitability is generated by the solubilization and vertical transport of $\mathrm{CO}_{2}$ in the $\mathrm{BZ}$ medium containing the surfactant sodium dodecyl sulfate (SDS). It causes an increase in proton concentration and, hence, in excitability of the BZ medium with the height of the reactor [27]. Furthermore, SDS suppresses or delays $\mathrm{CO}_{2}$ bubble formation [27-29], enhancing the observation of 3D patterns by optical tomography [5,18,27,30-33].

In this Letter, scroll waves were induced perpendicularly to the vertical gradient of excitability. Filaments that deviate only slightly from the horizontal orientation were found to develop a twist which starts from one edge of the filament and subsequently propagates along it. By contrast, filaments that are slightly U-shaped develop two twists that start from both edges of the filament and possess opposite senses of rotation (opposite handedness, i.e., opposite signs 
of the phase gradient along the filament). A schematic representation of these two cases is shown in Fig. 1 [34].

Experimental methods.-Experiments were performed using a ferroin-catalyzed BZ reaction in an agarose (type VII, Sigma) gel matrix, which suppresses hydrodynamical fluxes. The initial concentrations were $50 \mathrm{mM}$ malonic acid (Merck), $50 \mathrm{mM}$ sodium bromate (Merck), $210 \mathrm{mM}$ sulfuric acid (Fluka), and $0.5 \mathrm{mM}$ ferroin. Furthermore, $0.2 \mathrm{mM}$ SDS (Fluka) were added to the medium to retard or suppress $\mathrm{CO}_{2}$ bubble formation [27-29]. In twodimensional (2D) measurements, this recipe generated rigidly rotating spiral waves. The temperature was kept constant at $21 \pm 1^{\circ} \mathrm{C}$.

The reaction medium was placed in a cylindrical cuvette of $21 \mathrm{~mm}$ inner diameter and $25 \mathrm{~mm}$ height. A horizontal scroll wave was initiated by the partition method $[15,18]$ and monitored by optical tomography. During a rotation of the reaction cuvette by $180^{\circ} 2 \mathrm{D}$ projections were recorded every $1.8^{\circ}$, yielding a tomographic sample of 100 projections with a resolution of $0.1 \mathrm{~mm} \mathrm{pixel}^{-1}[18,27]$. A slicewise reconstruction of the samples was ensued by filtered backprojection of the ferriin concentration [33]. The reconstructed horizontal slices were rearranged to vertical slices, such that each vertical slice contained a spiral wave. To visualize the 3D structures, isoconcentration surfaces were calculated from the stack of horizontal slices [Figs. 2(a) and 2(d)].

The filament was extracted to study the dynamics of a scroll wave in detail. It is the central area of the scroll wave which is never excited by the wave front during one revolution of the scroll wave [35]. The phase along a filament is determined from the stack of vertical slices of the reconstructed data by placing a line at the tip of the spiral along the inner front of the wave. The phase angle between this line and a reference was measured.

Results.-Horizontal scroll waves were oriented perpendicularly to both, the long axis of the cylindrical reaction cuvette and a reaction-induced vertical $\mathrm{CO}_{2}$ gradient. (a)

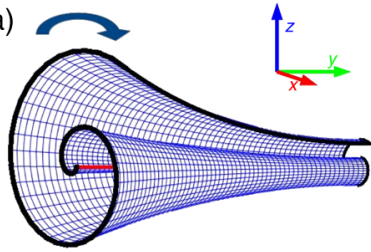

(b)

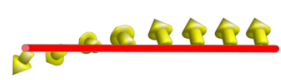

(c)

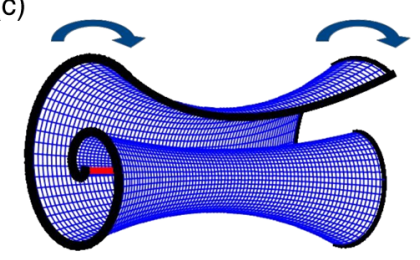

(d)

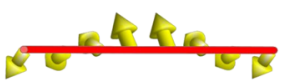

FIG. 1 (color online). Schematic drawing of a scroll wave that twists only from one side (a) and a scroll wave developing a pair of twists of opposite handedness at its two ends (c). The arrows show the domain where the scroll wave rotates faster, thus inducing the twist. In panels (b) and (d) the vectors and the line represent the twist of the filament and the filament itself, respectively. Note that the vectors are arranged symmetrically, reflecting a mirror symmetry of the scroll wave.
In the closed reactor dissolved $\mathrm{CO}_{2}$ was prevented from escaping to the atmosphere, such that it accumulated at the top of the reactor [27]. While scroll waves oriented exactly parallel to the horizontal remained unaffected by the gradient, as predicted in Ref. [17], filaments that were slightly bent at the boundaries were prone to twist.

Tilted scroll waves twist only at one end of the filament, namely the end that was bent upwards or downwards at the boundary [Fig. 2(a)]. No twist was formed at the other end when the filament was exactly attached perpendicularly to the boundary. The bent part of the filament is exposed to a domain of (slightly) higher excitability. The twist started at the end of the filament, propagated along it, but did not intersperse the entire scroll wave, so that a part remained untwisted [Figs. 2(a) and 2(c)]. Furthermore, the scroll was bent in the horizontal plane [Fig. 2(b)]. This was due to the initiation of the scroll, when the ends of the filament were not anchored exactly on the opposite sides of the reactor. Then the scroll wave can be considered as a segment of a scroll ring, which shrank due to its positive line tension $[23,24]$.
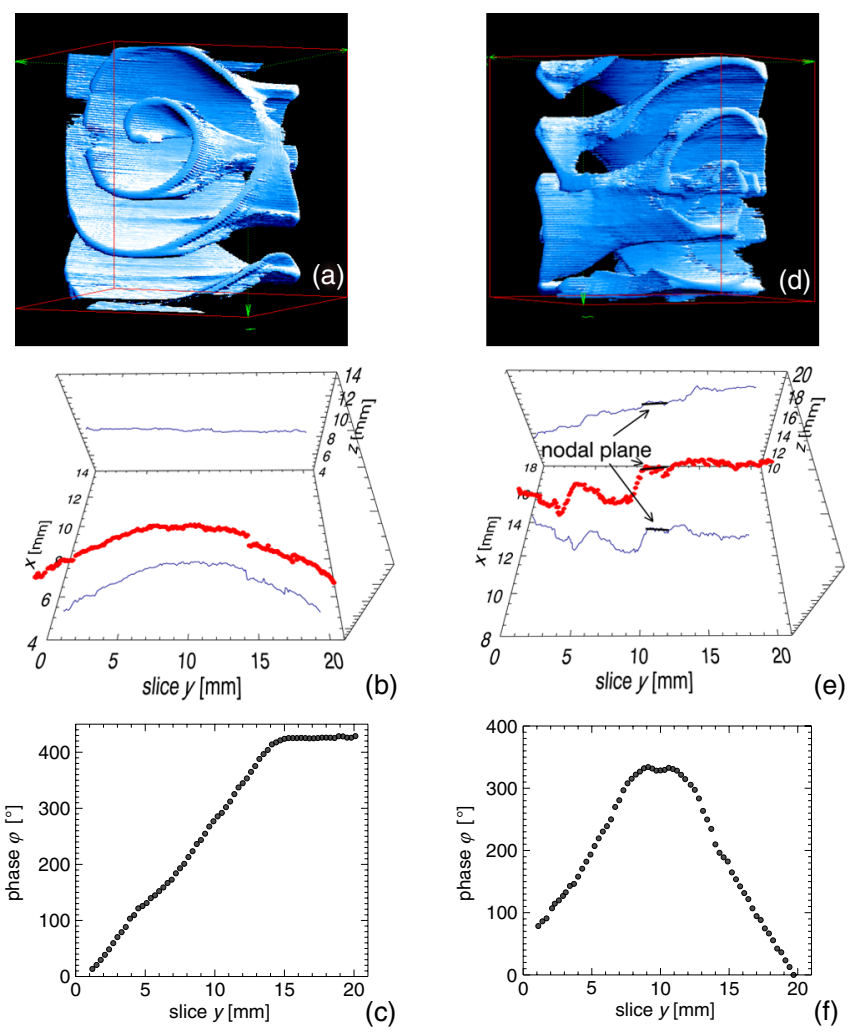

FIG. 2 (color online). Scroll waves (a)-(c) with a tilted and (d)-(f) a U-shaped filament at $t=418 \mathrm{~min}$. The isoconcentration surfaces are shown in (a) with sample dimensions of $21 \times 21 \times 23.1 \mathrm{~mm}^{3}$ and (d) $21 \times 21 \times 22 \mathrm{~mm}^{3}$. The corresponding reconstructions of the filaments (bold red dotted line) and the projections to the $x y$ and $y z$ planes (thin blue lines) were (b) tilted at one side and (e) U-shaped before starting to twist. The phase along the filament is shown for (c) the tilted filament and (f) the U-shaped filament. 
Scroll waves, whose filaments were slightly deformed upwards or downwards at the boundaries, i.e., U-shaped, became twisted from both ends of the filament [Figs. 2(d) and 2(f)]. The scroll wave of Fig. 2(d) was inclined with respect to the horizontal direction (by $16^{\circ}$ ), but its filament was slightly bent upwards at the lower-lying end. The twists at the two ends of the filament did not start simultaneously: The end which was closer to the top of the reaction cuvette began to twist first, whereas the lowerlying end of the filament started to develop a twist after a delay of $44 \mathrm{~min}$.

The two twists had an opposite sense of rotation. The twists were $35.1 \mathrm{deg} \mathrm{mm} \mathrm{mm}^{-1}$ and therefore $d \varphi / d y>0$ on the left side (lower-lying end) and $-40.3 \mathrm{deg} \mathrm{mm}^{-1}$ on the right side (higher lying end; $d \varphi / d y<0$ ) of the filament [Fig. 2(f)]. Both twists grew from the edges to the center of the filament, forming a nodal plane separating the twists from each other [Fig. 2(f)]. There, the scroll remained untwisted and the filament rectilinear, whereas the rest of the filament was twisted. The nodal plane is visible in the reconstruction of the filament and in its projections to the $x y$ and $y z$ planes [Fig. 2(e)].

Modeling.-We consider the scaled and dimensionless Oregonator [36] as a model of the BZ reaction. It consists of two reaction-diffusion equations, one equation describing the fast activator $u$ and the other the slow inhibitor $v$ :

$$
\begin{aligned}
\frac{\partial u}{\partial t} & =\frac{1}{\epsilon}\left(u(1-u)-f v \frac{u-q}{u+q}\right)+D_{u} \nabla^{2} u, \\
\frac{\partial v}{\partial t} & =u-v+D_{v} \nabla^{2} v
\end{aligned}
$$

where the small parameter $\epsilon$ controls the excitability of the medium and depends on the concentration of protons in the reactor, as $\epsilon \sim\left[H^{+}\right]^{-1}$ [37]. The variables $u$ and $v$ describe the dynamics of the concentrations of $\mathrm{HBrO}_{2}$ and the catalyst ferroin, respectively. To mimic the gradient of $\mathrm{CO}_{2}$ in the reactor, a vertical gradient of excitability was introduced in analogy to earlier work [27] by setting

$$
\epsilon(z)=\epsilon_{0} /(1+K z),
$$

where $z$ is the vertical coordinate and $K$ is a constant. In contrast to previous studies [27], a linear gradient of $\mathrm{CO}_{2}$ in the reactor was assumed.

To reproduce the cylindrical shape of the reactor with nonflux boundaries we have used a phase field method. We defined a phase field $\phi$, where $\phi=1$ inside and $\phi=0$ outside the reactor [27]. The chemical concentrations were renormalized to $u \rightarrow \phi u$ and $v \rightarrow \phi v$.

Simulations. - In the presence of a vertical gradient a perpendicular scroll wave remained straight and no twist was observed in the simulation. If half of the filament was initially bent upwards, the scroll wave twisted because of the difference in excitability produced by the gradient. The other side remained untwisted. The twist grew with time and the phase difference between both ends of the filament increased. After some time the scroll wave twisted [Fig. 3(a)], the filament remained curved [Fig. 3(b)], and the twist increased [Fig. 3(c)].

If both ends of the filament were bent upwards, the simulations showed that the scroll wave produced two twists of opposite handedness [Fig. 3(d)], as observed in the experiments. The filament was bent at both sides as shown in Fig. 3(e) and due to the symmetry of the initial condition the twists at both ends were identical [Fig. 3(f)]. The dynamics of the scroll waves in the simulations were in good agreement with the experiments.

Due to the twist and the cylindrical boundary conditions the filament curved into a piece of a scroll ring, which slowly shrank driven by the positive filament tension.

Twist evolution.-In the experiments, the twists on the left and right side of the nodal plane increased with time and the magnitude of the slopes was equal for a symmetrically twisted scroll wave [Fig. 4(a)]. For experiments with asymmetrically twisted scroll waves the nodal plane was not localized in the middle of the filament.

In Fig. 4(b) the evolution of the twist at both ends of the filament of the $\mathrm{U}$-shaped filament is plotted. The twist grew
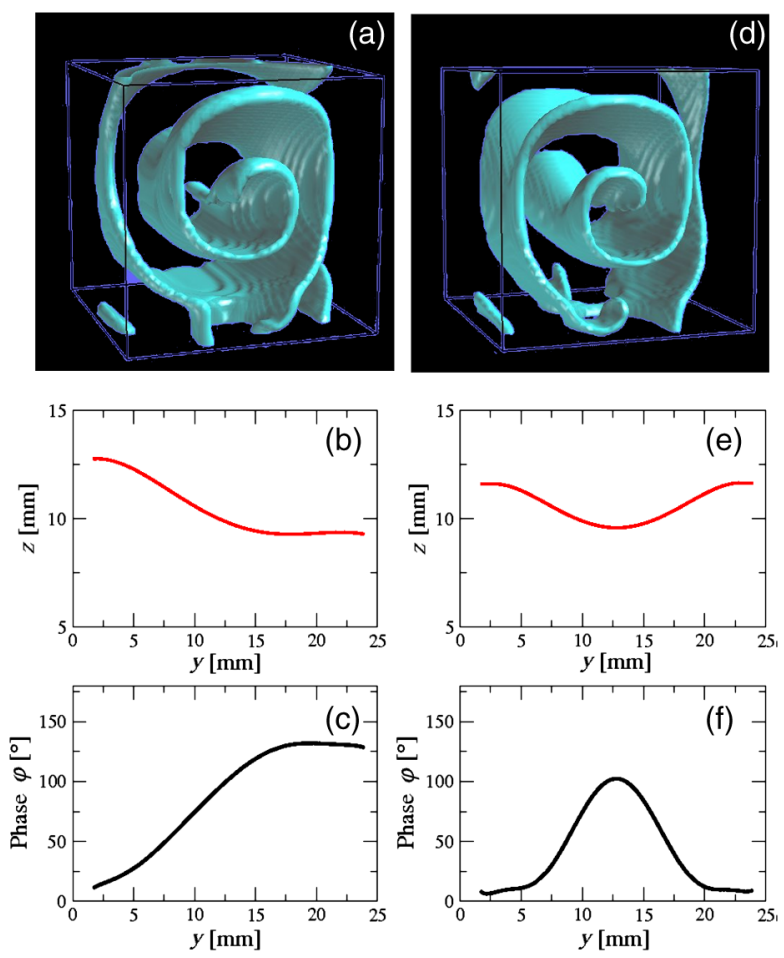

FIG. 3 (color online). Scroll waves obtained by numerical simulations with a filament (a)-(c) tilted at one side and (d)-(f) at both sides after $t=50 \mathrm{~min}$ in a system of $25 \times 25 \times$ $23 \mathrm{~mm}^{3}$. Isoconcentration surfaces for (a) a tilted and (d) a $U$-shaped scroll wave. Projection in the plane $y z$ of the (b) tilted and (e) U-shaped filament of the scroll wave. Phase of (c) the tilted and (f) U-shaped filament. Values of the parameters: $D_{u}=1, D_{v}=0.6, q=0.002, f=2, \epsilon_{0}=0.03$, and $K=0.065 \mathrm{~mm}^{-1}$. 

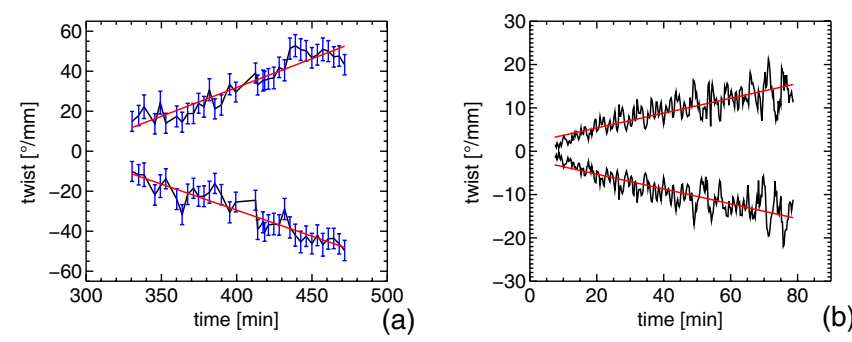

FIG. 4 (color online). Comparison of the evolution of the twists for the U-shaped filament (a) in the experiment and (b) in the simulation. The black curves represent the twists of different handedness of the scroll waves. Except for the sign, the slopes for both linear fits of the twist on the scroll wave are almost equal.

almost linearly with time at both sides in a symmetric way. Eventually, the filament in the simulation lost its initially bent shape due to the positive filament tension, and the twist stopped to grow for a simulation time up to $400 \mathrm{~min}$. The initial growth of the filament twist in the numerical simulations agreed qualitatively with that of the experiments, however, the twists observed in the experiments were larger by a factor of 2 as compared to the ones observed in numerical simulations.

Discussion.-In the $\mathrm{BZ}$ reaction $\mathrm{CO}_{2}$ develops as an undesired product. To suppress or delay the $\mathrm{CO}_{2}$ bubble formation SDS is added to the reaction. However, it was shown that SDS generates a vertical gradient of excitability [27]. The excitability increases linearly with the height of the reactor, whereas underneath the lid of the reactor it grows exponentially [27]. In the experiments reported here the scroll waves were localized almost in the center of the reactor and therefore in the approximately linear part of the gradient.

Increasing front velocities of the scroll wave at the top of the $\mathrm{BZ}$ medium are responsible for the development of a twist. Scroll waves lying almost perpendicular to this gradient can also develop a twist under certain conditions. Initially straight filaments, whose ends are perpendicular to the boundaries, do not twist and they drift perpendicularly to the gradient, as predicted in Ref. [17]. If the filament is initially bent, two cases can occur depending on the shape of the deformation: The scroll wave can develop either a one-sided twist [Figs. 2(a)-2(c)] or two regions of twist of opposite handedness that start from both ends of the filament [Figs. 2(d)-2(f)]. Therefore, the filament must be bent upwards or downwards directly at the boundaries of the cuvette (i.e., $U$-shaped) when the $\mathrm{CO}_{2}$ gradient is sufficiently strong to induce a twist in the scroll wave. The filament must find a shape representing a compromise between the positive line tension and the gradient [38]. The twists of opposite handedness are separated by a nodal plane, where the filament remains rectilinear and untwisted.

The experimentally observed scroll wave dynamics have been reproduced in numerical simulations using a
Oregonator model with a linear gradient of excitability [Fig. 3]. Small perturbations of the straight filament induce the formation of twist. On the other hand, the positive tension of the filament reduces the filament deformation. Both effects compete and the filament returns to a straight configuration, although the accumulation of twist persists and induces the deformation of the filament perpendicularly to the gradient. This asymptotic dynamics is not observed in the experiments, since the reaction medium becomes exhausted before the asymptotic state is reached. Eventually, it produces a piece of scroll ring which collapses.

The opposite twists at the two ends of the filament result from the mirror symmetry of the scroll wave [Figs. 1(c) and 1(d)]. While twisting, the wave fronts propagate faster and the scroll wave twists in the same direction in which the scroll rotates. Horizontal filaments which are bent in the middle but perpendicular to the boundaries were observed not to twist.

In summary, scroll waves carrying twists of opposite handedness are not "strange beasts" but a generic response of excitable media to a gradient of excitability. Our investigations show that the accumulated twists are very longlived. As a result, scroll waves in gradient fields can be expected to be prone to twist-induced instabilities as long as their filaments have a small component oriented in parallel to the gradient. Consequently, even moderate gradients may lead to irregular dynamics and scroll wave breakup. Our findings may also be of importance with regard to cardiac arrhythmias, since scroll waves with a pair of twists of opposite handedness may be generated in the heart between the endocardium and epicardium. These scrolls twist due to different propagation velocities in the different domains of the myocardium. Therefore, our findings may help in understanding the dynamics of some cardiac arrhythmias, and be of deep relevance for the development of more sophisticated methods to control (annihilate) such structures.

We thank the Graduiertenförderung des Landes Sachsen-Anhalt for financial support for Patricia Dähmlow. We acknowledge support by the DFG within the SFB 910 "Control of Self-Organizing Nonlinear Systems."

*marcus.hauser@ovgu.de

[1] A. T. Winfree, Science 266, 1003 (1994).

[2] A. T. Winfree, When Time Breaks Down: The ThreeDimensional Dynamics of Electrochemical Waves and Cardiac Arrhythmias (Princeton University Press, Princeton, NJ, 1987).

[3] F. H. Fenton, E. M. Cherry, H. M. Hastings, and S.J. Evans, Chaos 12, 852 (2002).

[4] A. T. Winfree, Science 181, 937 (1973).

[5] C. Luengviriya, U. Storb, M. J.B. Hauser, and S.C. Müller, Phys. Chem. Chem. Phys. 8, 1425 (2006). 
[6] C. Luengviriya, U. Storb, G. Lindner, S.C. Müller, M. Bär, and M. J. B. Hauser, Phys. Rev. Lett. 100, 148302 (2008).

[7] T. Bánsági and O. Steinbock, Phys. Rev. E 76, 045202 (2007).

[8] N. F. Otani, S. Luther, R. Singh, and J. Gilmour, F. Robert, Ann. Biomed. Eng. 38, 3112 (2010).

[9] S. Luther, F. H. Fenton, B. G. Kornreich, A. Squires, P. Bittihn, D. Hornung, M. Zabel, J. Flanders, A. Gladuli, L. Campoy, E. M. Cherry, G. Luther, G. Hasenfuss, V. I. Krinsky, A. Pumir, R.F. Gilmour, and E. Bodenschatz, Nature (London) 475, 235 (2011).

[10] M. Gotoh, T. Uchida, W. Fan, M. C. Fishbein, H. S. Karagueuzian, and P.S. Chen, Am. J. Physiol. 272, H107 (1997).

[11] F. J. Vetter and A. D. McCulloch, Prog. Biophys. Molec. Biol. 69, 157 (1998).

[12] M. Wellner, O. Berenfeld, J. Jalife, and A. M. Pertsov, Proc. Natl. Acad. Sci. U.S.A. 99, 8015 (2002).

[13] H. Verschelde, H. Dierckx, and O. Bernus, Phys. Rev. Lett. 99, 168104 (2007).

[14] C. Henze, E. Lugosi, and A. T. Winfree, Can. J. Phys. 68, 683 (1990).

[15] A.M. Pertsov, R.R. Aliev, and V.I. Krinsky, Nature (London) 345, 419 (1990).

[16] H. Henry and V. Hakim, Phys. Rev. E 65, 046235 (2002).

[17] S. Mironov, M. Vinson, S. Mulvey, and A. Pertsov, J. Phys. Chem. 100, 1975 (1996).

[18] U. Storb, C. R. Neto, M. Bär, and S.C. Müller, Phys. Chem. Chem. Phys. 5, 2344 (2003).

[19] A. Mikhailov, A. Panfilov, and A. Rudenko, Phys. Lett. 109A, 246 (1985).

[20] B. Echebarria, V. Hakim, and H. Henry, Phys. Rev. Lett. 96, 098301 (2006).
[21] T. Amemiya, P. Kettunen, S. Kadar, T. Yamaguchi, and K. Showalter, Chaos 8, 872 (1998).

[22] C. Zhang, H. Liao, and Q. Ouyang, J. Phys. Chem. B 110, 7508 (2006).

[23] C. Luengviriya and M. J. B. Hauser, Phys. Rev. E 77, 056214 (2008).

[24] C. Luengviriya, S. C. Müller, and M. J. B. Hauser, Phys. Rev. E 77, 015201 (2008).

[25] Z. Yang, S. Gao, Q. Ouyang, and H. Wang, Phys. Rev. E 86, 056209 (2012).

[26] M. Vinson, S. Mironov, S. Mulvey, and A. Pertsov, Nature (London) 386, 477 (1997).

[27] D. Kupitz, S. Alonso, M. Bär, and M. J. B. Hauser, Phys. Rev. E 84, 056210 (2011).

[28] A. Lázár, Z. Noszticzius, H.-D. Försterling, and Z. NagyUngvárai, Physica (Amsterdam) 84D, 112 (1995).

[29] A. Belmonte, J.-M. Flesselles, and Q. Ouyang, Europhys. Lett. 35, 665 (1996).

[30] B. Marts, T. Bánsági, and O. Steinbock, Europhys. Lett. 83, 30010 (2008).

[31] D. Kupitz and M. J. B. Hauser, Phys. Rev. E 86, 066208 (2012).

[32] A. T. Winfree, S. Caudle, G. Chen, P. McGuire, and Z. Szilagyi, Chaos 6, 617 (1996).

[33] D. Stock and S. C. Müller, Physica (Amsterdam) 96D, 396 (1996).

[34] See Supplemental Material at http://link.aps.org/ supplemental/10.1103/PhysRevLett.110.234102 for a schematic movie of the twist generation at the two ends.

[35] S. C. Müller, T. Plesser, and B. Hess, Science 230, 661 (1985).

[36] J. J. Tyson and P. C. Fife, J. Chem. Phys. 73, 2224 (1980).

[37] R. J. Field, Scholarpedia 2, 1386 (2007).

[38] G. Rousseau, H. Chate, and R. Kapral, Chaos 18, 026103 (2008). 